\title{
Synthesis and antiviral activity of a series of new cyclohexenyl nucleosides
}

\author{
Ping Gu${ }^{1}$, Jordi Morral ${ }^{1}$, Jing Wang ${ }^{1}$, Jef Rozenski ${ }^{1}$, Roger Busson ${ }^{1}$, Arthur Van Aerschot ${ }^{1}$, \\ Erik De Clercq ${ }^{2}$ and Piet Herdewijn ${ }^{1 *}$ \\ 'Laboratory of Medicinal Chemistry and 'Laboratory of Virology and Chemotherapy, Rega Institute for \\ Medical Research, Katholieke Universiteit Leuven, Leuven, Belgium
}

*Corresponding author: Tel: +32 1633 7387; Fax: +32 1633 7340; E-mail: piet.herdewijn@rega.kuleuven.ac.be

\begin{abstract}
A series of new cyclohexenyl nucleosides is synthesized by coupling the heterocyclic bases with a protected cyclohexenyl precursor under Mitsunobu conditions. The compounds were evaluated for their antiviral and cytostatic activity. Pronounced activity against herpes simplex virus
\end{abstract}

type 1 and type 2 was observed for the 2,6diaminopurine analogue.

Keywords: synthesis, antiviral, cyclohexenyl nucleosides

\section{Introduction}

The development of new nucleoside analogues as antiviral agents has remained an attractive research field. Due to their hydrolytic stability, carbocyclic nucleosides have taken a particular place in the design process of new antiviral agents (Marquez, 1996). Most of these compounds are cyclopentane derivatives and less work has been done on conformationally more rigid carbocyclic analogues such as cyclohexane nucleosides. Some of these cyclohexane nucleosides were synthesized in the past but they were devoid of antiviral activity (Schaeffer et al., 1964, 1968; Pérez-Pérez, 1995; Mikhailov et al., 1996; Maurinsh et al., 1997). However, the more flexible cyclohexene nucleosides look more promising. Indeed, introduction of a double bond into the cyclohexane ring could facilitate the phosphorylation of the nucleoside analogue and their eventual incorporation in DNA and, hence, lead to antiviral activity (Wang et al., 2000). A cyclohexene ring is more flexible than a cyclohexane ring and is more prone to conformational changes that might be needed for substrate/inhibitor recognition during enzymatic reactions.

Therefore, cyclohexenyl guanine (Cycl-G) 1 and cyclohexenyl adenine (Cycl-A) $\mathbf{2}$ were synthesized and their biological activities were investigated (Wang et al., 2000). In particular, D-cyclohexenyl-G has found to exhibit potent and selective activity against herpes viruses [herpes simplex virus type 1 (HSV-1) and type $2(\mathrm{HSV}-2)$, varicella-zoster virus (VZV), cytomegalovirus (CMV)], in analogy with that of the known antiviral drugs acyclovir and ganciclovir (Wang et al., 2000). This activity could be explained by the intercellular phosphorylation of $\mathrm{Cycl}-\mathrm{G}$ to its triphosphate in virus-infected cells, as deduced from the low activity of cyclohexenyl $\mathrm{G}$ against thymidine kinasedeficient $\left(\mathrm{TK}^{-}\right)$viral strains (Figure 1$)$.
These results prompted us to synthesize other cyclohexenyl nucleosides (3-10) in order to study the effects of base modification on antiviral activity and toxicity. We recently reported a straightforward procedure for obtaining the racemic $\mathbf{1}$ using as the key step a Diels-Alder reaction (Wang et al., 2001). The compounds envisaged here were obtained analogously as racemic mixtures, as separation of the $(+)$ and $(-)$ enantiomers proved tedious. In case of a positive biological evaluation, the synthesis of the separate enantiomers could be envisaged.

\section{Materials and methods}

\section{Chemistry}

For all reactions, analytical grade solvents were used. All moisture-sensitive reactions were carried out in ovendried glassware $\left(100^{\circ} \mathrm{C}\right)$ under a nitrogen atmosphere. Anhydrous solvent 1,4-dioxane was refluxed on sodium/benzophenone and distilled. Melting points were determined in capillary tubes with a Büchi SMP-20 cap. apparatus and were uncorrected. ${ }^{1} \mathrm{H}$ NMR was determined with a Varian Unity $500 \mathrm{MHz}$ spectrometer with tetramethylsilane (TMS) as internal standard for ${ }^{1} \mathrm{H}$ NMR spectra and a $200 \mathrm{MHz}$ Varian Gemini apparatus was used for ${ }^{13} \mathrm{C}$ NMR determination with DMSO- $\mathrm{d}_{6}$ (39.6 ppm) or $\mathrm{CDCl}_{3}$ (76.9 ppm) as internal standard for the ${ }^{13} \mathrm{C}$ NMR spectra (s, singlet; d, doublet; dd, double doublet; t, triplet; br s, broad singlet; br d, broad doublet; $\mathrm{m}$, multiplet). Exact mass measurements were performed on a quadrupole time-of-flight mass spectrometer (QTof-2, Micromass, Manchester, UK) equipped with a standard electrospray-ionization (ESI) interface; samples were infused in $i-\mathrm{PrOH} / \mathrm{H}_{2} \mathrm{O} 1: 1$ at $3 \mu \mathrm{l} / \mathrm{min}$. Precoated 

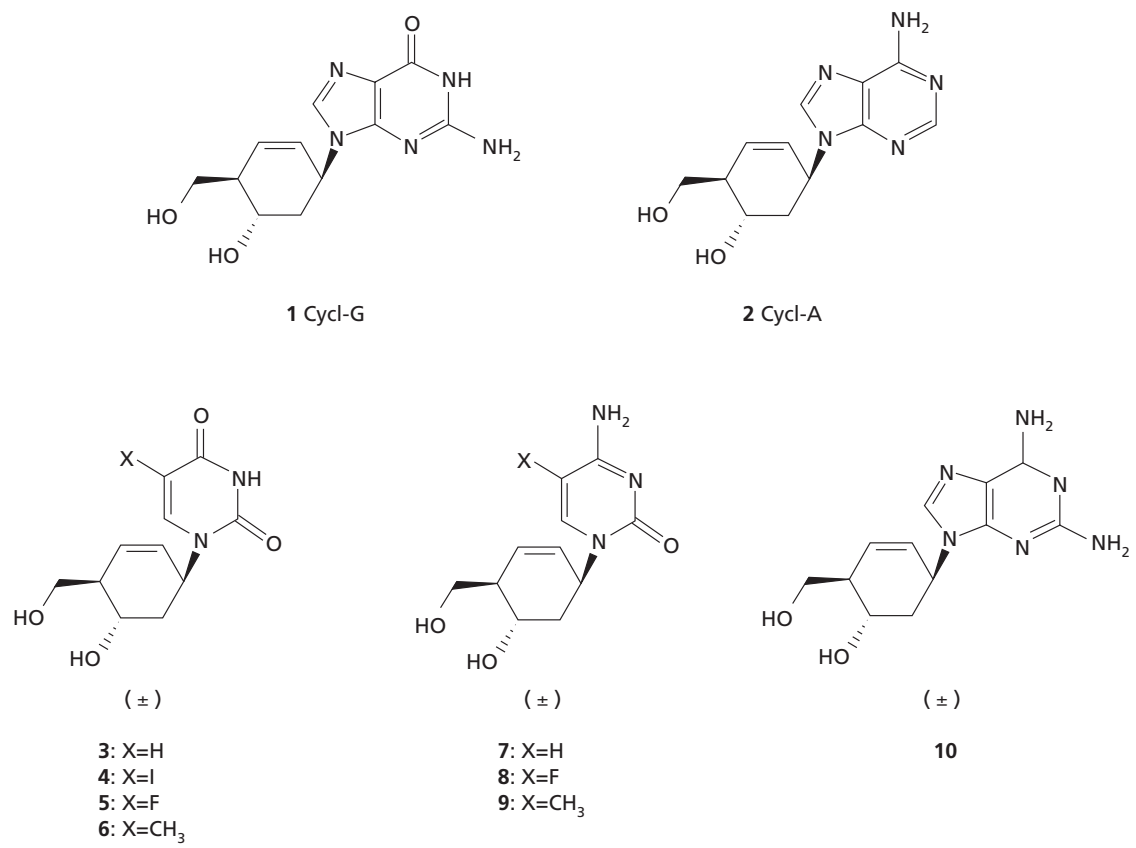

$( \pm)$

10

aluminum sheets (Fluka Silica gel/TLC-cards, $254 \mathrm{~nm}$ ) were used for TLC; the spots were examined with UV light, or sprayed with sulfuric acid/anisaldehyde or $1 \%$ potassium permanganate solution. Column chromatography was performed on ICN silica gel 63-200 60 A. Elemental analyses were done at the University of Konstanz, Germany, and were in agreement with calculated values within $0.4 \%$ error margin. The names of the compounds accorded to the rules of IUPAC and were checked with a nomenclature program (ACD-Labs, Version 4.08, Sept. 1999, Adv. Chem. Dev., Inc., Toronto, Canada).

( \pm )-(4aR,7R, 8aS)-2-phenyl-4a, 7,8,8a-tetrahydro-4H1,3-benzodioxin-7-ol (11)

The preparation of this compound has been described previously (Wang et al., 2001).

(土)-1-[(1S,4R,5S)-5-hydroxy-4-(hydroxymethyl)-2-cyclohexen-1-yl]-2,4(1H,3H)-pyrimidinedione (3)

A suspension of $1.73 \mathrm{~g}$ ( $8 \mathrm{mmol})$ of $\mathrm{N}^{3}$-benzoyluracil (12), $0.93 \mathrm{~g}$ (4 mmol) of $( \pm)-(4 \mathrm{aR}, 7 \mathrm{R}, 8 \mathrm{aS})-2-$ Phenyl4a,7,8,8a-tetrahydro-4H-1,3-benzodioxin-7-ol (11), 1.15 $\mathrm{g}(8 \mathrm{mmol})$ sodium benzoate and $2.62 \mathrm{~g}(10 \mathrm{mmol})$ triphenylphosphine in $100 \mathrm{ml}$ of anhydrous dioxane was stirred under nitrogen. A solution of $1.60 \mathrm{ml}(10 \mathrm{mmol})$ of diethylazodicarboxylate (DEAD) in $40 \mathrm{ml}$ anhydrous dioxane was slowly added over a period of $3 \mathrm{~h}$. The mixture was further stirred overnight at room temperature and filtered, and the filtrate was distilled in vacuo to remove the solvent. The residue was dissolved in $100 \mathrm{ml}$ of methanol saturated with ammonia and stirred overnight at room temperature. Evaporation and coevaporation with methanol left colourless oil that was purified on silica gel EtOAc/n-Hexane 5-40\% $\quad\left(\mathrm{R}_{\mathrm{f}}=0.4\right)$. Compound 21 was obtained as a white solid $0.51 \mathrm{~g}(1.6$ mmol, yield 36\%).

Compound 21 (300 mg, $0.91 \mathrm{mmol}$ ) was treated with 20 $\mathrm{ml}$ of $80 \%$ trifluoroacetic acid solution at room temperature for 2 days. After evaporation and coevaporation with toluene and methanol, the residue was dissolved in $10 \mathrm{ml}$ water and extracted with ether. The water layer was concentrated and the resulting white solid was purified by column chromatography $\left(\mathrm{CH}_{3} \mathrm{OH} / \mathrm{EtOAc} 0-8 \%, \mathrm{R}_{\mathrm{f}}=0.3\right)$. Crystallization from $\mathrm{CH}_{3} \mathrm{OH} / \mathrm{EtOAc}$ afforded 3 as a white crystal (138 mg, $0.55 \mathrm{mmol}$, overall yield 23\%).

mp. $171^{\circ} \mathrm{C} ;{ }^{1} \mathrm{H}$ NMR (DMSO-d $)$ $\delta 1.78$ (m, 2H, H-2', $2^{\prime \prime}, 2.08$ (dt, 1H, J=2.5 Hz, $\left.5.5 \mathrm{~Hz}, \mathrm{H}-4^{\prime}\right), 3.51$ (m, 2H, $\left.\mathrm{CH}_{2} \mathrm{OH}\right), 3.72$ (m, 1H, H-3'), 4.70 (br s, $1 \mathrm{H},-\mathrm{CH}_{2} \mathrm{OH}$ ), 4.78 (br s, $\left.1 \mathrm{H}, 3^{\prime}-\mathrm{OH}\right), 5.10\left(\mathrm{~m}, 1 \mathrm{H}, \mathrm{H}-1^{\prime}\right), 5.54$ (d, $1 \mathrm{H}$, $\mathrm{J}=8.0 \mathrm{~Hz}, \mathrm{H}-5$ ), 5.61 (ddd, $1 \mathrm{H}, \mathrm{J}=2.5 \mathrm{~Hz}, 3.5 \mathrm{~Hz}, 10.0 \mathrm{~Hz}$, H-6'), 5.98 (ddd, 1H, J=2.0 Hz, 3.0 Hz, 10.2 Hz, H-5'), 7.44 (d, 1H, J=8.0 Hz, H-6), 11.28 (br s, $1 \mathrm{H},-\mathrm{NH}$ ); ${ }^{13} \mathrm{C}$ NMR (DMSO-d $)$ \& $34.6\left(\mathrm{C}-2^{\prime}\right), 46.0\left(\mathrm{C}-4^{\prime}\right), 50.1\left(\mathrm{C}-1^{\prime}\right)$, 61.7 (- $\left.\underline{\mathrm{CH}}_{2} \mathrm{OH}\right), 63.1\left(\mathrm{C}-3^{\prime}\right), 101.1(\mathrm{C}-5), 125.3\left(\mathrm{C}-6^{\prime}\right)$, 134.8 (C-5'), 142.6 (C-6), 151.1 (C-2), 163.6 (C-4) ppm. HRMS calcd. for $\mathrm{C}_{11} \mathrm{H}_{15} \mathrm{~N}_{2} \mathrm{O}_{4}(\mathrm{M}+\mathrm{H})^{+}: 239.1032$, found 239.1019. 
( \pm )-1-[(1S,4R,5S)-5-hydroxy-4-(hydroxymethyl)-2-cyclohexen-1-yl]-5-iodo-2,4(1H,3H)-pyrimidinedione (4)

Starting with $0.46 \mathrm{~g}$ ( $2 \mathrm{mmol})$ of compound 11, $1.36 \mathrm{~g}$ (4 mmol) of $\mathrm{N}^{3}$-benzoyl-5-iodouracil (13), $1.0 \mathrm{~g}$ (4 mmol) triphenylphosphine, $0.58 \mathrm{~g}(4 \mathrm{mmol})$ of sodium benzoate and $0.72 \mathrm{ml}$ (4 mmol) of DEAD in $20 \mathrm{ml}$ anhydrous dioxane, using the same procedure as described for 3 , after purification by column chromatography $\left(\mathrm{CH}_{3} \mathrm{OH} / \mathrm{CH}_{2} \mathrm{Cl}_{2}\right.$ $\left.0-10 \%, R_{f}=0.5\right), 203 \mathrm{mg}(0.56 \mathrm{mmol}$, overall yield $29 \%)$ of compound 4 was obtained.

mp. $207^{\circ} \mathrm{C} ;{ }^{1} \mathrm{H}$ NMR (DMSO-d $) \delta 1.81$ (m, 2H, H-2', $\left.2^{\prime \prime}\right), 2.10\left(\mathrm{~m}, 1 \mathrm{H}, \mathrm{H}-4^{\prime}\right), 3.59\left(\mathrm{~m}, 2 \mathrm{H},-\mathrm{CH}_{2} \mathrm{OH}\right), 3.75(\mathrm{~m}$, $\left.1 \mathrm{H}, \mathrm{H}-3^{\prime}\right), 4.72-4.83$ (m, 2H, $\left.-\mathrm{CH}_{2} \mathrm{OH}, 3^{\prime}-\mathrm{OH}\right), 5.08$ (m, 1H, H-1'), 5.65 (ddd, 1H, J=2.2 Hz, $3.9 \mathrm{~Hz}, 10.0 \mathrm{~Hz}, \mathrm{H}-$ 6'), 6.03 (ddd, $1 \mathrm{H}, \mathrm{J}=1.7 \mathrm{~Hz}, 3.2 \mathrm{~Hz}, 10.0 \mathrm{~Hz}, \mathrm{H}-5^{\prime}$ ), 7.86 (s, 1H, H-6), 11.61 (br s, $1 \mathrm{H},-\mathrm{NH}$ ); ${ }^{13} \mathrm{C}$ NMR (DMSO$\left.\mathrm{d}_{6}\right) \delta 34.5\left(\mathrm{C}-2^{\prime}\right), 46.1\left(\mathrm{C}-4^{\prime}\right), 50.7\left(\mathrm{C}-1^{\prime}\right), 61.3(-$ $\left.\mathrm{CH}_{2} \mathrm{OH}\right), 62.8\left(\mathrm{C}-3^{\prime}\right), 68.2(\mathrm{C}-5), 124.9\left(\mathrm{C}-6^{\prime}\right), 135.4$ (C5'), 146.7 (C-6), 150.6 (C-2), 160.7 (C-4) ppm. HRMS calcd. for $\mathrm{C}_{11} \mathrm{H}_{14} \mathrm{IN}_{2} \mathrm{O}_{4}(\mathrm{M}+\mathrm{H})^{+}$: 365.0000, found 365.0013 .

\section{(土)-5-fluoro-1-[(1S,4R,5S)-5-hydroxy-4-(hydrox- ymethyl)-2-cyclohexen-1-yl]-2,4(1H,3H)-pyrimidine- dione (5)}

Following the same procedure as described for 3 , and starting with $0.93 \mathrm{~g}$ (4 mmol) of $11,1.87 \mathrm{~g}(8 \mathrm{mmol})$ of $\mathrm{N}^{3}$ benzoyl-5-fluorouracil (14), $2.62 \mathrm{~g}(10 \mathrm{mmol})$ of triphenylphosphine, $1.15 \mathrm{~g}(8 \mathrm{mmol})$ of sodium benzoate and $1.60 \mathrm{ml}(10 \mathrm{mmol})$ of DEAD in $40 \mathrm{ml}$ anhydrous dioxane, $550 \mathrm{mg}$ of compound 23 was obtained. After purification by column chromatography $\left(\mathrm{CH}_{3} \mathrm{OH} / \mathrm{CH}_{2} \mathrm{Cl}_{2}, 0-10 \%\right.$, $\mathrm{R}_{\mathrm{f}}=0.3$ ), the desired fluorouracil derivative $\mathbf{5}$ was obtained as a white crystal in $44 \%$ yield ( $112 \mathrm{mg}, 0.44 \mathrm{mmol}$ ).

mp. $208^{\circ} \mathrm{C} ;{ }^{1} \mathrm{H}$ NMR (DMSO-d $)_{6} \delta$ 1.74-1.84 (m, 2H, H-2', 2' ), 2.08 (m, 1H, H-4'), 3.50-3.58 (ddd, 2H, J=5.1 $\left.\mathrm{Hz}, 10.5 \mathrm{~Hz}, 22.5 \mathrm{~Hz},-\mathrm{CH}_{2} \mathrm{OH}\right), 3.79$ (m, 1H, H-3'), $4.71\left(\mathrm{t}, 1 \mathrm{H}, \mathrm{J}=5.3 \mathrm{~Hz},-\mathrm{CH}_{2} \mathrm{O} \underline{\mathrm{H}}\right), 4.76(\mathrm{~d}, 1 \mathrm{H}, \mathrm{J}=4.4 \mathrm{~Hz}$, $\left.3^{\prime}-\mathrm{OH}\right), 5.09$ (m, 1H, H-1'), 5.62 (ddd, 1H, J=2.2 Hz, 3.4 $\mathrm{Hz}, 10.0 \mathrm{~Hz}, \mathrm{H}-6^{\prime}$ ), 5.95 (ddd, 1H, J=1.9 Hz, 3.2 Hz, 10.0 $\mathrm{Hz}, \mathrm{H}-5^{\prime}$ ), 7.73 (d, 1H, J=7.1 Hz, H-6), 11.81 (br s, 1H, $\mathrm{NH}) ;{ }^{13} \mathrm{C}$ NMR (DMSO-d 6 ) $\delta 34.1$ (C-2'), 46.0 (C-4'), $50.5\left(\mathrm{C}-1^{\prime}\right), 61.5\left(-\underline{\mathrm{H}}_{2} \mathrm{OH}\right), 62.9\left(\mathrm{C}-3^{\prime}\right), 125.1$ (C-6'), 126.8127 .5 (C-6), 135.2 (C-5'), 137.4142 .0 (C-5), 149.7 (C-2), 157.6 (C-4) ppm. HRMS calcd. for $\mathrm{C}_{11} \mathrm{H}_{14} \mathrm{FN}_{2} \mathrm{O}_{4}$ $(\mathrm{M}+\mathrm{H})^{+}:$257.0938, found 257.0900.

(土)-1-[(1S,4R,5S)-5-hydroxy-4-(hydroxymethyl)-2-cyclohexen-1-yl]-5-methyl-2,4(1H,3H)-pyrimidinedione (6)

To a solution of $0.83 \mathrm{~g}(3.6 \mathrm{mmol})$ of $( \pm)-(4 \mathrm{aR}, 7 \mathrm{R}, 8 \mathrm{aS})-2-$ Phenyl-4a,7,8,8a-tetrahydro-4H-1,3-benzodioxin-7-ol (11), $1.64 \mathrm{~g}$ (7.2 mmol) of $\mathrm{N}^{3}$-benzoylthymine (15), $1.04 \mathrm{~g}$
(7.2 mmol) of sodium benzoate and $1.88 \mathrm{~g}(7.2 \mathrm{mmol})$ of triphenylphosphine in $100 \mathrm{ml}$ of anhydrous dioxane, a solution of $1.41 \mathrm{ml}(7.2 \mathrm{mmol})$ of DIAD in $30 \mathrm{ml}$ was drop wise added under nitrogen environment. The mixture was kept stirring overnight at room temperature. The reaction was filtered and evaporated. The crude $\mathbf{2 0}$ was directly treated with $100 \mathrm{ml}$ saturated ammonia/methanol solution for $6 \mathrm{~h}$. After evaporation and coevaporation with methanol, a colourless oil of thymine analogue $\mathbf{2 4}$ was obtained and it was further treated with $40 \mathrm{ml} 80 \%$ trifluoroacetic acid water solution for 2 days. Workup purification on silica gel $\left(\mathrm{CH}_{3} \mathrm{OH} / \mathrm{CH}_{2} \mathrm{Cl}_{2} 0-10 \%, \mathrm{R}_{\mathrm{f}}=0.3\right)$ and recrystallization from $\mathrm{CH}_{3} \mathrm{OH} / \mathrm{EtOAc}$ yielded a white crystal 6 (144 mg, $0.57 \mathrm{mmol}$, overall yield 16\%).

mp. $227^{\circ} \mathrm{C} ;{ }^{1} \mathrm{H}$ NMR (DMSO-d $)$ ס $1.73(\mathrm{~d}, 3 \mathrm{H}, \mathrm{J}=1.0$ $\mathrm{Hz},-\mathrm{CH}_{3}$ ), 1.77 (br t, 2H, J=5.7 Hz, H-2', 2'), 2.09 (m, $\left.1 \mathrm{H}, \mathrm{H}-4^{\prime}\right), 3.54$ (m, 2H, $\left.-\underline{\mathrm{CH}}_{2} \mathrm{OH}\right), 3.79$ (m, 1H, H-3'), $4.76\left(\mathrm{t}, 1 \mathrm{H}, \mathrm{J}=5.2 \mathrm{~Hz},-\mathrm{CH}_{2} \mathrm{O} \underline{\mathrm{H}}\right), 4.80(\mathrm{~d}, 1 \mathrm{H}, \mathrm{J}=4.2 \mathrm{~Hz}$, $\left.3^{\prime}-\mathrm{OH}\right), 5.10\left(\mathrm{~m}, 1 \mathrm{H}, \mathrm{H}-1^{\prime}\right), 5.60$ (ddd, 1H, J=2.2 Hz, 3.4 $\mathrm{Hz}, 10.0 \mathrm{~Hz}, \mathrm{H}-6^{\prime}$ ), 5.96 (ddd, 1H, J=2.1 Hz, 3.0 Hz, 10.1 Hz, H-5'), 7.31 (d, 1H, J=1.2 Hz, H-6), 11.31 (br s, 1H, $\mathrm{NH}) ;{ }^{13} \mathrm{C}$ NMR (DMSO-d $)$ \& $12.2\left(-\mathrm{CH}_{3}\right), 34.4\left(\mathrm{C}-2^{\prime}\right)$, 46.1 (C-4'), $49.6\left(\mathrm{C}-1^{\prime}\right), 61.7\left(-\underline{\mathrm{CH}}_{2} \mathrm{OH}\right), 63.1\left(\mathrm{C}-3^{\prime}\right)$, 108.7 (C-5), 125.7 (C-6'), 134.6 (C-5'), 138.4 (C-6), 151.1 (C-2), 164.3 (C-4) ppm. HRMS calcd. for $\mathrm{C}_{12} \mathrm{H}_{17} \mathrm{~N}_{2} \mathrm{O}_{4}(\mathrm{M}+\mathrm{H})^{+}: 253.1188$, found 253.1180.

\section{(土)-4-Amino-1-[(1S,4R,5S)-5-hydroxy-4-(hydrox-} ymethyl)-2-cyclohexen-1-yl]-2(1H)-pyrimidinone (7)

A premixed solution of 1,2,4-triazole (309 mg, $4.48 \mathrm{mmol}$ ) and phosphoroxychloride $(120 \mu \mathrm{l}, 1.28 \mathrm{mmol})$ in $30 \mathrm{ml}$ dried pyridine was added to $210 \mathrm{mg}(0.64 \mathrm{mmol})$ of $( \pm)-1-$ [(4aR,7S,8aS)-2-phenyl-4a,7,8,8a,-tetrahydro-4H-1,3benzodioxin-7-yl]-4-amino-2 $(1 \mathrm{H})$-pyrimidinone (21). The mixture was stirred at room temperature. After $16 \mathrm{~h}$, the mixture was cooled to $0^{\circ} \mathrm{C}$ in an ice bath and ammonia gas was bubbled for $12 \mathrm{~min}$ and the reaction was left for 10 min further at room temperature. Evaporation and coevaporation with toluene and methanol, a yellow syrup was obtained which was further treated with $80 \%$ trifluoroacetic acid solution $(30 \mathrm{ml})$ for 2 days at room temperature. The reaction mixture was concentrated, and coevaporated with toluene and methanol. The residue was chromatographed on silica gel $\left(\mathrm{CH}_{3} \mathrm{OH} / \mathrm{CH}_{2} \mathrm{Cl}_{2}\right.$ 5-20\%, $\left.\mathrm{R}_{\mathrm{f}}=0.5\right)$ to yield 7 (62.4 mg, $0.26 \mathrm{mmol}$, overall yield $\left.41 \%\right)$ as a light yellow solid.

mp. $129^{\circ} \mathrm{C} ;{ }^{1} \mathrm{H}$ NMR (DMSO-d $)$ $\delta 1.78$ (m, 2H, H-2', $2^{\prime \prime}$ ), 2.08 (m, 1H, H-4'), 3.48-3.56 (ddd, $2 \mathrm{H}, \mathrm{J}=5.4 \mathrm{~Hz}$, $\left.10.6 \mathrm{~Hz}, 21.4 \mathrm{~Hz},-\mathrm{CH}_{2} \mathrm{OH}\right), 3.68$ (m, 1H, H-3'), 4.67 (br s, $\left.1 \mathrm{H},-\mathrm{CH}_{2} \mathrm{O} \underline{\mathrm{H}}\right), 4.74$ (br s, $\left.1 \mathrm{H}, 3^{\prime}-\mathrm{OH}\right), 5.15$ (m, 1H, H1'), 5.58 (ddd, 1H, J=2.2 Hz, 3.7 Hz, $10.0 \mathrm{~Hz}, \mathrm{H}-6^{\prime}$ ), 5.82 (d, 1H, J=7.5 Hz, H-5), 6.00 (ddd, 1H, J=2.0 Hz, 3.2 Hz, $\left.10.0 \mathrm{~Hz}, \mathrm{H}-5^{\prime}\right), 7.58$ (d, 1H, J=7.3 Hz, H-6), 7.64, 8.02 (br 
d, $\left.2 \mathrm{H},-\mathrm{NH}_{2}\right) ;{ }^{13} \mathrm{C}$ NMR (DMSO-d $)$ \& $34.8\left(\mathrm{C}-2^{\prime}\right), 46.2$ $\left(\mathrm{C}-4^{\prime}\right), 51.0\left(\mathrm{C}-1^{\prime}\right), 61.7\left(-\underline{\mathrm{CH}}_{2} \mathrm{OH}\right), 62.9\left(\mathrm{C}-3^{\prime}\right), 93.5$ (C5), $125.2\left(\mathrm{C}-6^{\prime}\right), 134.9\left(\mathrm{C}-5^{\prime}\right), 144.7$ (C-6), 152.8 (C-2), $163.3(\mathrm{C}-4)$ ppm. HRMS calcd. for $\mathrm{C}_{11} \mathrm{H}_{16} \mathrm{~N}_{3} \mathrm{O}_{3}(\mathrm{M}+\mathrm{H})^{+}$: 238.1192, found 238.1179.

\section{(士)-4-Amino-5-fluoro-1-[(1S,4R,5S)-5-hydroxy-4- (hydroxymethyl)-2-cyclohexen-1-yl]-2(1H)-pyrimidi- none (8)}

Following the procedure used for preparation of 7 , and started from $172 \mathrm{mg}(0.5 \mathrm{mmol})$ of $( \pm)-1-[(4 \mathrm{aR}, 7 \mathrm{~S}, 8 \mathrm{aS})-$ 2-phenyl-4a,7,8,8a-tetrahydro-4H-1,3-benzodioxin-7yl]-5-fluoro-2,4 (1H,3H)-pyrimidinone (23), 1,2,4-triazole (242 mg, $3.5 \mathrm{mmol}$ ), phosphoroxychloride (93 $\mu \mathrm{l}, 1$ $\mathrm{mmol}$ ) in $30 \mathrm{ml}$ dry pyridine, the 5-fluorocytosine analogue was obtained $(59.9 \mathrm{mg}, 0.2 \mathrm{mmol})$ in an overall yield of $47 \%$.

mp. $264^{\circ} \mathrm{C} ;{ }^{1} \mathrm{H}$ NMR (DMSO-d $)$ o 1.71-1.82 (m, 2H, $\left.\mathrm{H}-2^{\prime}, 2^{\prime \prime}\right), 2.05$ (m, 1H, H-4'), 3.56 (m, 2H, $\left.-\mathrm{CH}_{2} \mathrm{OH}\right)$, 3.70 (m, 1H, H-3'), 4.68 (t, 1H, J=5.1 Hz, $\left.-\mathrm{CH}_{2} \mathrm{O} \underline{\mathrm{H}}\right), 4.70$ (d, $1 \mathrm{H}, \mathrm{J}=4.4 \mathrm{~Hz}, 3^{\prime}-\mathrm{OH}$ ), 5.09 (m, 1H, H-1'), 5.60 (ddd, 1H, J=2.2 Hz, 3.9 Hz, 10.0 Hz, H-6'), 5.98 (m, 1H, H-5'),

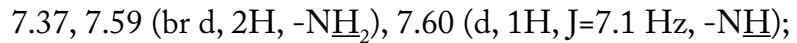
${ }^{13} \mathrm{C}$ NMR (DMSO-d $)$ \& $35.0\left(\mathrm{C}-2^{\prime}\right), 46.3$ (C-4'), 51.0 (C$\left.1^{\prime}\right), 61.4\left(-\mathrm{CH}_{2} \mathrm{OH}\right), 62.5\left(\mathrm{C}-3^{\prime}\right), 125.4\left(\mathrm{C}-6^{\prime}\right), 127.5(\mathrm{C}-$ 6), 127.9 (C-5'), 135.4 (C-5), 154.0 (C-2), 157.4 (C-4) ppm. HRMS calcd. for $\mathrm{C}_{11} \mathrm{H}_{15} \mathrm{FN}_{3} \mathrm{O}_{3}(\mathrm{M}+\mathrm{H})^{+}:$: 256.1097, found 256.1080 .

\section{(士)-4-Amino-1-[(1S,4R,5S)-5-hydroxy-4-(hydrox-} ymethyl)-2-cyclohexen-1-yl]-5-methyl-2(1H)-pyrimidinone (9)

Starting with $( \pm)-1-[(4 \mathrm{aR}, 7 \mathrm{~S}, 8 \mathrm{aS})-2$-phenyl-4a,7,8,8atetrahydro-4H-1,3-benzodioxin-7-yl]-5-methyl2,4(1H,3H)-pyrimidinone (24) (148 $\mathrm{mg}, 0.44 \mathrm{mmol})$, 1,2,4-triazole (240 mg, $3.48 \mathrm{mmol}$ ), phosphoroxychloride $(81 \mu \mathrm{l}, 0.86 \mathrm{mmol})$ and $15 \mathrm{ml}$ dry pyridine, using the same procedure as described for 7 , and purified on silica gel $\left(\mathrm{CH}_{3} \mathrm{OH} / \mathrm{CH}_{2} \mathrm{Cl}_{2}\right.$ 5-20\%, $\left.\mathrm{R}_{\mathrm{f}}=0.4\right)$, a white solid was obtained, which was crystallized from $\mathrm{CH}_{2} \mathrm{Cl}_{2} / \mathrm{CH}_{3} \mathrm{OH}$ (60 mg, $0.2 \mathrm{mmol}$, overall yield 55\%).

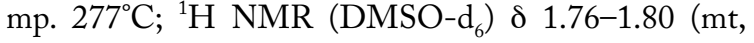
2H, H-2', 2' ), 1.83 (s, 3H, $-\mathrm{CH}_{3}$ ), 2.09 (m, 1H, H-4'), $3.54\left(\mathrm{~m}, 2 \mathrm{H},-\mathrm{CH}_{2} \mathrm{OH}\right), 3.70\left(\mathrm{~m}, 1 \mathrm{H}, \mathrm{H}-3^{\prime}\right), 4.70-4.78$ (br, $\left.2 \mathrm{H},-\mathrm{CH}_{2} \mathrm{O} \underline{\mathrm{H}}, 3^{\prime}-\mathrm{O} \underline{\mathrm{H}}\right), 5.08\left(\mathrm{~m}, 1 \mathrm{H}, \mathrm{H}-1^{\prime}\right), 5.58$ (ddd, $1 \mathrm{H}, \mathrm{J}=2.1 \mathrm{~Hz}, 3.6 \mathrm{~Hz}, 10.0 \mathrm{~Hz}, \mathrm{H}-6^{\prime}$ ), 5.96 (ddd, $1 \mathrm{H}$, $\left.\mathrm{J}=2.6 \mathrm{~Hz}, 3.0 \mathrm{~Hz}, 10.2 \mathrm{~Hz}, \mathrm{H}-5^{\prime}\right), 7.48$ (d, 1H, J=1.2 Hz, H-6), 8.07-8.57 (br d, 2H, - $\left.\underline{\mathrm{H}}_{2}\right) ;{ }^{13} \mathrm{C} \mathrm{NMR}$ (DMSO-d ${ }_{6}$ ) ठ $12.7\left(-\underline{\mathrm{CH}}_{3}\right), 34.4\left(\mathrm{C}-2^{\prime}\right), 46.2\left(\mathrm{C}-4^{\prime}\right), 50.9\left(\mathrm{C}-1^{\prime}\right), 61.7$ $\left(-\mathrm{CH}_{2} \mathrm{OH}\right), 62.9\left(\mathrm{C}-3^{\prime}\right), 101.3(\mathrm{C}-5), 125.4\left(\mathrm{C}-6^{\prime}\right), 135.0$ (C-5'), 142.6 (C-6), 151.6 (C-2), 162.1 (C-4) ppm. HRMS calcd. for $\mathrm{C}_{12} \mathrm{H}_{18} \mathrm{~N}_{3} \mathrm{O}_{3}(\mathrm{M}+\mathrm{H})^{+}: 252.1348$, found 252.1330 .
( \pm )-(1S,2R,5S)-5-(2,6-diamino-9H-purin-9-yl)-2(hydroxymethyl)-3-cyclohexen-1-ol (10)

To a mixture of $( \pm)-(4 \mathrm{aR}, 7 \mathrm{R}, 8 \mathrm{aS})-2$-phenyl-4a,7,8,8atetrahydro-4H-1,3-benzodioxin-7-ol (11) $500 \mathrm{mg}(2.2$ mmol), 2-amino-6-chloropurine (821 $\mathrm{mg}, 4.8 \mathrm{mmol}$ ) and triphenylphosphine $(1.15 \mathrm{~g}, 4.4 \mathrm{mmol})$ in dry dioxane 30 $\mathrm{ml}$ under $\mathrm{N}_{2}$, at room temperature, was slowly added a solution of DIAD $(0.86 \mathrm{ml}, 4.4 \mathrm{mmol})$ in dry dioxane 20 $\mathrm{ml}$ over a period of $3 \mathrm{~h}$. The reaction mixture was stirred at room temperature for 2 days, filtered and concentrated. The residue was submitted to column chromatography $\left(\mathrm{CH}_{3} \mathrm{OH} / \mathrm{CH}_{2} \mathrm{Cl}_{2} 1 \%, \mathrm{R}_{\mathrm{f}}=0.3\right)$, a mixture of compound 25 and triphenylphosphine oxide was obtained. Due to the difficult separation, we decided to deprotect 25 before final purification.

A solution of impure compound 25 (about $1.4 \mathrm{~g}$ ) in 100 $\mathrm{ml}$ methanol saturated with ammonia is heated in a Parr pressure reactor for $8 \mathrm{~h}$ at $100^{\circ} \mathrm{C}$. After evaporation, the obtained residue was purified by flash chromatography $\left(\mathrm{CH}_{3} \mathrm{OH} / \mathrm{CH}_{2} \mathrm{Cl}_{2}\right.$ 0-8\%, $\left.\mathrm{R}_{\mathrm{f}}=0.3\right)$ to give $270 \mathrm{mg}(0.74$ $\mathrm{mmol}$ ) of 26 as yellow syrup. The benzylidene moiety was removed with $80 \%$ trifluoroacetic acid solution. After evaporation and coevaporation with toluene and methanol, the residue was purified by flash chromatography $\left(\mathrm{CH}_{3} \mathrm{OH} / \mathrm{CH}_{2} \mathrm{Cl}_{2}\right.$ 5-20\%, $\left.\mathrm{R}_{\mathrm{f}}=0.5\right)$ to give diaminopurine analogue 10 (107 mg, $0.39 \mathrm{mmol}$, overall yield 18\%), which was crystallized from $\mathrm{CH}_{2} \mathrm{Cl}_{2} / \mathrm{CH}_{3} \mathrm{OH}$ to give a white crystal.

mp. $268^{\circ} \mathrm{C} ;{ }^{1} \mathrm{H}$ NMR (DMSO-d $) \delta 1.87(\mathrm{~m}, 1 \mathrm{H}, \mathrm{H}-$ $\left.2^{\prime}\right), 1.97$ (dt, 1H, J=4.0 Hz, $\left.13.2 \mathrm{~Hz}, \mathrm{H}-2^{\prime \prime}\right), 2.12$ (m, 1H, $\left.\mathrm{H}-4^{\prime}\right), 3.52-3.66\left(\mathrm{~m}, 3 \mathrm{H},-\mathrm{CH}_{2} \mathrm{OH}, \mathrm{H}-3^{\prime}\right), 4.70(\mathrm{t}, 1 \mathrm{H}$, $\left.\mathrm{J}=5.2 \mathrm{~Hz},-\mathrm{CH}_{2} \mathrm{OH}\right), 4.76$ (d, $\left.1 \mathrm{H}, \mathrm{J}=5.4 \mathrm{~Hz}, 3^{\prime}-\mathrm{O} \underline{\mathrm{H}}\right), 4.99$ (m, $1 \mathrm{H}, \mathrm{H}-1^{\prime}$ ), 5.77 (ddd, $1 \mathrm{H}, \mathrm{J}=2.5 \mathrm{~Hz}, 3.9 \mathrm{~Hz}, 10.0 \mathrm{~Hz}$, H-6'), 5.83 (br s, 2H, $-\underline{\mathrm{N}}_{2}$ ), 5.98 (ddd, $1 \mathrm{H}, \mathrm{J}=1.6 \mathrm{~Hz}, 2.9$ $\mathrm{Hz}, 9.9 \mathrm{~Hz}, \mathrm{H}-5^{\prime}$ ), 6.68 (br s, 2H, $-\underline{\mathrm{N}}_{2}$ ) 7.60 (s, $1 \mathrm{H}, \mathrm{H}-$ $8) ;{ }^{13} \mathrm{C}$ NMR (DMSO-d 6 ) $\delta 35.9\left(\mathrm{C}-2^{\prime}\right), 46.6\left(\mathrm{C}-4^{\prime}\right), 47.9$ $\left(\mathrm{C}-1^{\prime}\right), 61.6\left(-\underline{\mathrm{CH}}_{2} \mathrm{OH}\right), 62.8\left(\mathrm{C}-3^{\prime}\right), 113.7(\mathrm{C}-5), 125.2$ (C-6'), $133.7\left(\mathrm{C}-5^{\prime}\right), 136.1$ (C-8), 151.5 (C-4), 156.4 (C6) 160.5 (C-2) ppm. HRMS calcd. for $\mathrm{C}_{12} \mathrm{H}_{17} \mathrm{~N}_{6} \mathrm{O}_{2}$ $(\mathrm{M}+\mathrm{H})^{+}:$277.1413, found 277.1416.

\section{Virology}

Antiviral activity determinations against herpesviruses were performed in either $\mathrm{E}_{6} \mathrm{SM}$ or HEL cell cultures as previously described (De Clercq et al., 1980). The origin of the viruses, HSV-1 (strains KOS, F and McIntyre), TK ${ }^{-} \mathrm{HSV}-1$ (strain KOS ACVr), HSV-2 (strains G, 196 and Lyons), VZV (strains OKA and YS), $\mathrm{TK}^{-} \mathrm{VZV}$ (strains $07-1$ and YS-R), vaccinia virus (VV), vesicular stomatitis virus (VSV) and CMV (strains AD169 and Davis) have been reported (De Clercq et al., 1986). The assays for evaluating activity 
against human herpesvirus 6 (HHV-6) have been described (De Clercq et al., 2001). The cytotoxicity measurements were based on microscopically visible alteration of normal cell morphology $\left(\mathrm{E}_{6} \mathrm{SM}\right)$ or inhibition of normal cell growth (HEL) as previously described (De Clercq et al., 1981).

\section{Results}

( \pm )-(4aR,7R,8aS)-2-phenyl-4a,7,8,8a-tetrahydro-4H-1,3benzodioxin-7-ol (11) is the key intermediate for the synthesis of cyclohexenyl nucleoside. A straightforward approach to synthesize compound $\mathbf{1 1}$ was recently reported (Wang et al., 2001). This involved a Diels-Alder cycloaddition reaction to build up the six-membered ring skeleton, a Fraser-Reid reductive rearrangement and protection of the diol with a benzylidene group. This approach was followed for the synthesis of 11, which was then used for base introduction.

For the synthesis of the uracil analogue 3, a Mitsunobu reaction was employed by using 1 eq. of compound 11, 2 eq. of $\mathrm{N}^{3}$-benzoyluracil 12, 2 eq. of triphenylphosphine $\left(\mathrm{Ph}_{3} \mathrm{P}\right)$, 2 eq. of sodium benzoate ( $\mathrm{PhCOONa}$ ), and 2 eq. of diethyl azodicarboxylate (DEAD) or diisopropyl azodicarboxylate (DIAD) in dioxane (Figure 2). The benzoyl group of $\mathbf{1 7}$ was removed by treatment with saturated ammonia/methanol and the benzylidene group was deprotected with $80 \%$ trifluoroacetic acid at room temperature. The uracil derivative 3 was obtained in $23 \%$ yield and, likewise, following the same procedure, starting from 13, 14 and 15, compounds 4, 5 and 6 were obtained in 29, 44 and 16\% overall yield, respectively.
Mitsunobu reaction using either $\mathrm{N}^{3}$-benzoylated thymine or uracil, or $\mathrm{N}$-benzoylcytosine afforded mainly the $\mathrm{O}^{2}$-substituted nucleosides. Only by addition of sodium benzoate the desired pyrimidine analogues could be obtained in moderate yield. The Mitsunobu conditions used here were somewhat different (Varasi et al., 1987; Hughes et al., 1988) from the standard conditions (Jenny et al., 1991), as we were unable to obtain the desired $\mathrm{N}^{1}$ substituted pyrimidine nucleoside analogues using these standard conditions.

The cytosine, 5-methylcytosine and 5-fluorocytosine analogues 7, 8 and 9 were obtained from their uracil counterparts (21, 23 and 24, respectively) via the 4-triazolylpyrimidinone intermediates (Krug et al., 1989) (Figure 3). Therefore, compounds 21, 23 and 24 were treated with phosphorous oxychloride and 1, 2, 4-triazole, and subsequently treated with bubbling ammonia gas through the reaction mixture for $10-15 \mathrm{~min}$. After chromatographic purification, the benzylidene group was removed with $80 \%$ trifluoroacetic acid solution to yield 7, 8 and 9 in overall yield of 15, 17 and 20\%, respectively (starting from compound 11).

2-Amino-6-chloropurine was introduced under Mitsunobu conditions to afford 25. This compound could be separated from its N-7 substituted isomer using column chromatography. Treatment of 25 with methanol saturated with ammonia in a Parr pressure reactor at $100^{\circ} \mathrm{C}$ (Verheggen et al., 1995) gave, after removal of the benzylidene moiety, the desired 2,6-diaminopurine derivative 10 in combined yield of $18 \%$ (Figure 4).

Figure 2. Synthesis of compounds 3, 4, 5 and 6

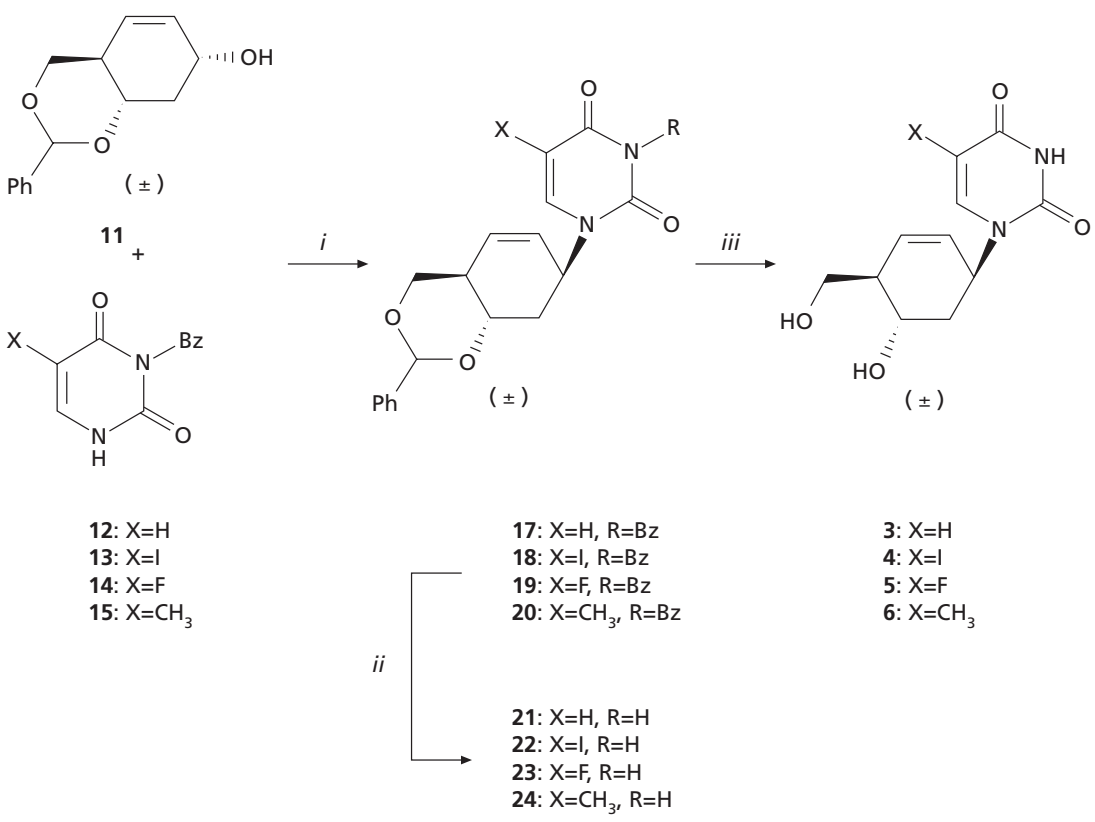

i) $\mathrm{Ph}_{3} \mathrm{P}, \mathrm{PhCOONa}, \mathrm{DEAD}$ or DIAD, Dioxane, r.t. 2 days; ii) $\mathrm{NH}_{3} / \mathrm{CH}_{3} \mathrm{OH}, 12 \mathrm{~h}$; iii) $\mathrm{TFA} / \mathrm{H}_{2} \mathrm{O}(80 \%)$. 


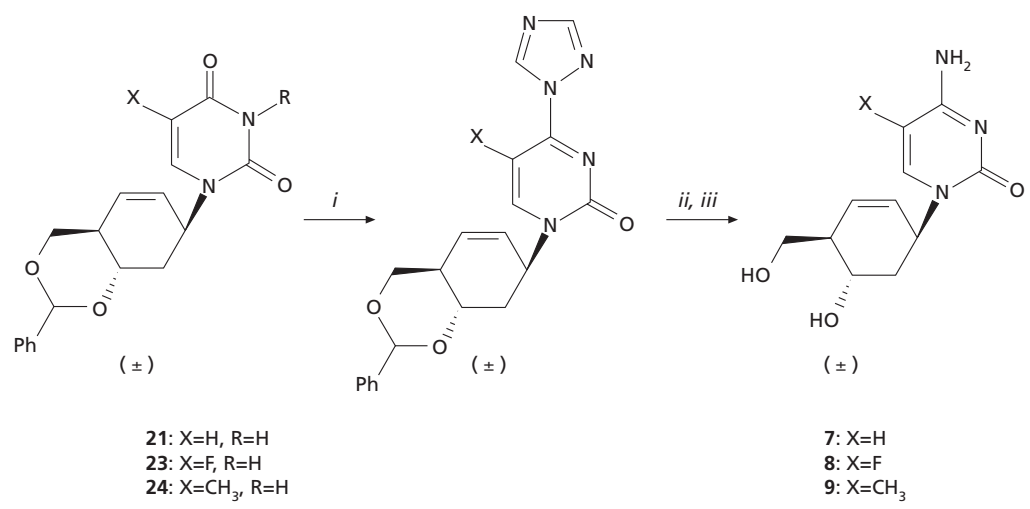

i) $\mathrm{POCl}_{3}, 1,2,4-1 \mathrm{H}$-triazole, pyridine; ii) $\mathrm{NH}_{3}$; iii) $\mathrm{TFA} / \mathrm{H}_{2} \mathrm{O}(80 \%)$.

\section{Discussion}

The newly synthesized compounds 4, 7, 8, 9 and 10 were investigated for their inhibitory effect on the cytopathogenicity of HSV-1, TK $\mathrm{TK}^{-} \mathrm{HS}-1, \mathrm{HSV}-2, \mathrm{VV}$ and VSV in human embryonic lung (HEL) cell cultures. Compounds 3 , 5 and $\mathbf{6}$ were tested against HSV-1, HSV-2 and VSV in human embryonic skin muscle $\left(\mathrm{E}_{6} \mathrm{SM}\right)$ fibroblast cell cultures (Table 1). The antiviral activity was compared with that of known and approved antiviral drugs, one with a pyrimidine base moiety (brivudin) and two with a purine base moiety (acyclovir, ganciclovir). The sources of the viruses and the methodology used to monitor antiviral activity have been described previously (De Clercq et al., 1980, 1986).

While compounds 3-5 did not display any meaningful activity, the analogues 6-10 demonstrated significant activity against HSV-1 and HSV-2. Of these compounds, 10 effected a $50 \%$ reduction of the cytopathogenicity induced by HSV-1 at a concentration of $1.4 \mu \mathrm{M}$. However, none of them approached the activity level of the reference compounds, brivudin, acyclovir or ganciclovir. The activity of the deoxycytidine analogues 7, 8 and 9 against
HSV-1 and HSV-2 was very similar. The diaminopurine derivative $\mathbf{1 0}$ is more potent against $\mathrm{HSV}-1$ than against $\mathrm{HSV}-2$. As the compounds were less active against the $\mathrm{TK}^{-}$acyclovir-resistant $\left(\mathrm{ACV}^{\mathrm{r}}\right)$ strain, intracellular phosphorylation by the virus-induced thymidine kinase must play an important role in their metabolic activation. Compounds were not active against VV at $210 \mu \mathrm{M}$. None of the compounds proved cytotoxic at a concentration up to $1000 \mu \mathrm{M}$, as monitored by microscopically detectable alteration of normal cell morphology. Compounds $\mathbf{3}$ and $\mathbf{6}$ were also evaluated for their activity against VZV and CMV. However, they exhibited no inhibitory effect on the cytopathogenicity of $\mathrm{VZV}$ (whether $\mathrm{TK}^{+}$or $\mathrm{TK}^{-}$) or $\mathrm{CMV}$ in HEL cells (data not shown). Compounds 3, 4 and 8-10 were not active against human herpesvirus type $6 \mathrm{~A}$ or $6 \mathrm{~B}$ in human T-lymphoblast HSB-2 and Molt-3 cells (De Clercq et al., 2001) at concentrations up to $20 \mu \mathrm{M}$ (data not shown).

From these results it is clear that only the diaminoguanine derivative, which can be considered as a precursor of cyclohexenyl-G, is of interest because of its moderate antiHSV activity. Further research is needed to unravel its

Figure 4. Synthesis of compound 10

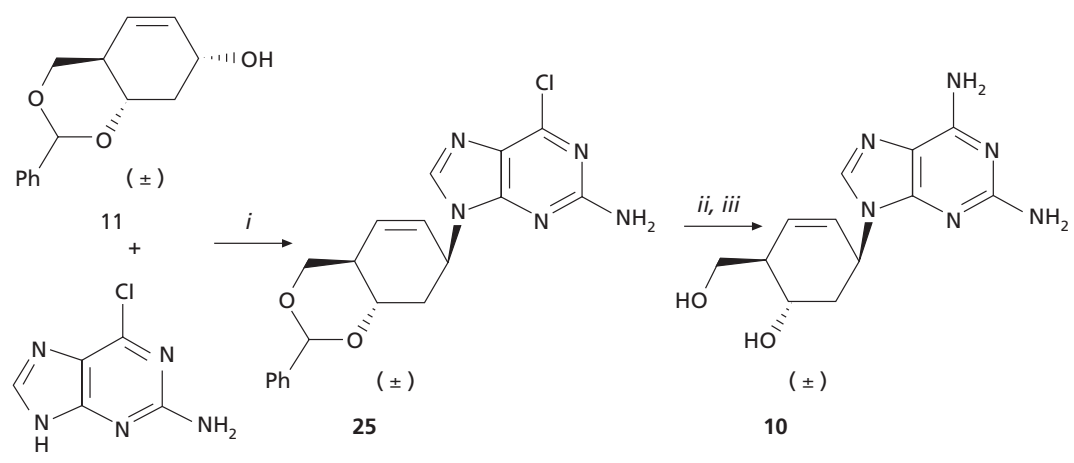

i) $\mathrm{Ph}_{3} \mathrm{P}$, PhCOONa, DIAD, Dioxane, r.t. 2 days; ii) $\mathrm{NH}_{3} / \mathrm{CH}_{3} \mathrm{OH}$, Parr bomb, $100^{\circ} \mathrm{C}, 14 \mathrm{~h}$; iii) $\mathrm{TFA} / \mathrm{H}_{2} \mathrm{O}(80 \%)$. 
Table 1. Antiviral activity against HSV-1, HSV-2, VV and VSV and cytotoxicity of compound 3-10

\begin{tabular}{|c|c|c|c|c|c|c|c|c|c|c|c|}
\hline \multicolumn{12}{|c|}{$\mathrm{MIC}(\mu \mathrm{M})$} \\
\hline Compound & 3 & 4 & 5 & 6 & 7 & 8 & 9 & 10 & D-Cycl G * & Acyclovir & Ganciclovir \\
\hline Cell virus & $\mathrm{E}_{6} \mathrm{SM}$ & HEL & $\mathrm{E}_{6} \mathrm{SM}$ & $\mathrm{E}_{6} \mathrm{SM}$ & HEL & HEL & HEL & HEL & $\mathrm{E}_{6} \mathrm{SM}$ & HEL & HEL \\
\hline HSV-1 (KOS) & 1007 & $>220$ & 936 & 190 & 40 & 63 & 191 & 1.4 & 0.01 & 0.4 & 0.02 \\
\hline HSV-1 (F) & ND§ & 220 & ND & ND & 13 & 38 & 64 & 2.3 & 0.01 & 0.6 & $<0.01$ \\
\hline HSV-1 (McIntyre) & ND & 659 & ND & ND & 202 & 38 & 191 & 6.9 & 0.01 & 0.6 & 0.01 \\
\hline HSV-1 (TK- KOS ACV') & 1007 & 1098 & ND & ND & 67 & 940 & 955 & 174 & 1.37 & 213 & 3.1 \\
\hline HSV-2 (G) & 1007 & 659 & $>312$ & 190 & 40 & 13 & 64 & 35 & 0.18 & 0.4 & $<0.01$ \\
\hline HSV-2 (196) & ND & 659 & ND & ND & 67 & 38 & 191 & 35 & 0.25 & 0.4 & 0.03 \\
\hline HSV-2 (Lyons) & ND & 659 & ND & ND & 67 & 188 & 191 & 35 & 0.25 & 0.4 & 0.01 \\
\hline VV & $>1679$ & $>220$ & $>312$ & $>1585$ & 1011 & $>1567$ & $>1592$ & 869 & ND & $>1775$ & $>392$ \\
\hline VSV & $>1679$ & $>220$ & $>312$ & $>1585$ & $>1686$ & $>1567$ & $>1592$ & $>290$ & ND & $>1775$ & $>392$ \\
\hline MCC & $>1679$ & $\geq 1098$ & $\geq 1561$ & $>1585$ & $>1686$ & $>1567$ & $>1592$ & $\geq 1448$ & $>1442$ & $>1775$ & $>392$ \\
\hline
\end{tabular}

MIC, minimum inhibitory concentration, or concentration required to reduce virus-induced cytopathogenicity by $50 \%$; $\mathrm{MCC}$, minimal cytotoxic concentration, or concentration required to cause a microscopically detectable alteration of normal cell morphology.

* Literature data taken from reference (Wang et al., 2000). ND, not determined.

mode of action and to evaluate the individual $(+)$ and $(-)$ enantiomers of compound $\mathbf{1 0 .}$

\section{Acknowledgements}

We thank A Marchand and I Lagoja for their helpful suggestions and $\mathrm{L}$ Baudemprez for NMR analysis. We also thank $A$ Van Lierde and F De Meyer for their excellent technical assistance and C Biernaux for her dedicated editorial help.

\section{References}

De Clercq E, Balzarini J, Torrence PF, Mertes MP, Schmidt CL, Shugar D, Barr PJ, Jones AS, Verhelst G \& Walker RT (1981)

Thymidylate synthetase as a target enzyme for the inhibitory activity of 5-substituted 2'-deoxyuridines on mouse leukemia L-1210 cell growth. Molecular Pharmacology 19:321-330.

De Clercq E, Descamps J, Verhelst G, Walker RT, Jones AS, Torrence PF \& Shugar D (1980) Comparative efficacy of antiherpes drugs against different strains of herpes simplex virus. Journal of Infectious Diseases 141:562-574.

De Clercq E, Hol A, Rosenberg I, Sakuma I, Balzarini J \& Maudgal P (1986) A novel selective broad-spectrum anti-DNA virus agent. Nature 323:464-467.

De Clercq E, Naesens L, De Bolle L, Schols D, Zhang Y \& Neyts J (2001) Antiviral agents active against human herpesviruses HHV6, HHV-7 and HHV-8. Reviews in Medical Virology 11:381-395.

Hughes DL, Reamer RA, Bergan JJ \& Grabowski EJJ (1988) A mechanistic study of the Mitsunobu esterification reaction. Journal of the American Chemical Society 110:6487-6491.

Jenny TF, Previsani N \& Benner SA (1991) Carbocyclic analogs of nucleosides via modified Mitsunobu reactions. Tetrahedron Letters 32:7029-7032.

Krug A, Schmidt S, Lekschars J, Lemke K \& Cech D (1989)

Transformation von uridin-zu cytidinderivaten durch selektive aminierung. (Transformation of uridine derivatives into cytidine via selective amination.) Journal für Praktische Chemie 331:835-842.
Marquez VE (1996) Carbocyclic nucleosides. In Advances in Antiviral Drug Design, vol. 2; pp. 89-146. Edited by E De Clercq. London: JAI Press.

Maurinsh Y, Schraml J, De Winter H, Blaton N, Peeters O, Lescrinier E, Rozenski J, Van Aerschot A, De Clercq E, Busson R \& Herdewijn P (1997) Synthesis and conformational study of 3hydroxy-4-(hydroxymethyl)-L-cyclohexanyl purines and pyrimidines. Journal of Organic Chemistry 62:2861-2871.

Mikhailov SN, Blaton N, Rozenski J, Balzarini J, De Clercq E \& Herdewijn P (1996) Use of cyclohexene epoxides in the preparation of carbocyclic nucleosides. Nucleosides, Nucleotides \& Nucleic Acids 15:867-878.

Pérez-Pérez M-J, Rozenski J, Busson R \& Herdewijn P (1995) Application of the Mitsunobu-type condensation reaction to the synthesis of phosphonate derivatives of cyclohexenyl and cyclohexanyl nucleosides. Journal of Organic Chemistry 60:1531-1537.

Schaeffer HJ, Kaistha KK \& Chakraborti SK (1964) Enzyme inhibitors II. Synthesis of trans-3-(6-substituted-9-purinyl)cyclohexanols as adenosine deaminase inhibitors. Journal of Pharmaceutical Science 53:1371-1374.

Schaeffer HJ \& Vince R (1968) Enzyme inhibitors XIX. The synthesis of some 1-hydroxy-2-hydroxymethyl-4-(6-substituted-9purinyl)cyclohexanes as nucleoside analogues. Journal of Medicinal Chemistry 11:15-20.

Varasi M, Walker KAM \& Maddox ML (1987) A revised mechanism for the Mitsunobu reaction. Journal of Organic Chemistry 52:4235-4238.

Verheggen I, Van Aerschot A, Van Meervelt L, Rozenski J, Wiebe L, Snoeck R, Andrei G, Balzarini J, Claes P, De Clercq E \& Herdewijn P (1995) Synthesis biological evaluation, and structure analysis of a series of new 1,5-anhydrohexitol nucleosides. Journal of Medicinal Chemistry 38:826-835.

Wang J, Froeyen M, Hendrix C, Andrei G, Snoeck R, De Clercq E \& Herdewijn P (2000) The cyclohexene ring system as a furanose mimic: synthesis and antiviral activity of both enantiomers of cyclohexenylguanine. Journal of Medicinal Chemistry 43:736-745.

Wang J, Morral J, Hendrix C \& Herdewijn P (2001) A straightforward stereoselective synthesis of D- and L-5-hydroxy-4-hydroxymethyl-2-cyclohexenylguanine. Journal of Organic Chemistry 66:8478-8482. 